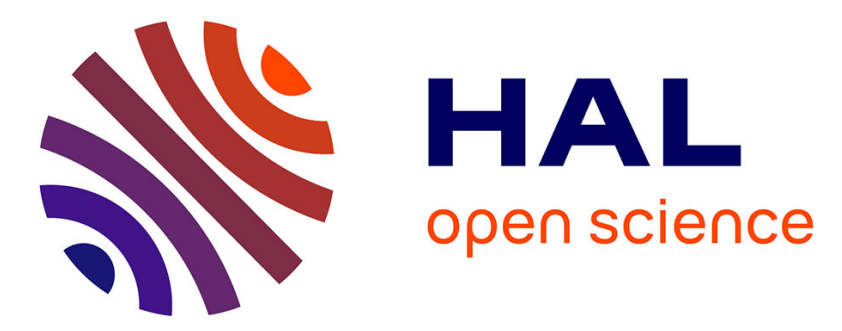

\title{
Argumentaire pour une utilisation plus large de la photochimiothérapie extracorporelle chez l'enfant
}

E. Merlin, J. Kanold, Dalil Hannani, D. Terral, M. D’incan, F. Deméocq

\section{To cite this version:}

E. Merlin, J. Kanold, Dalil Hannani, D. Terral, M. D'incan, et al.. Argumentaire pour une utilisation plus large de la photochimiothérapie extracorporelle chez l'enfant. Archives de Pédiatrie, 2010, 17 (9), pp.1327-1337. 10.1016/j.arcped.2010.06.017 . hal-02047427

\section{HAL Id: hal-02047427 \\ https://hal.science/hal-02047427}

Submitted on 17 Dec 2020

HAL is a multi-disciplinary open access archive for the deposit and dissemination of scientific research documents, whether they are published or not. The documents may come from teaching and research institutions in France or abroad, or from public or private research centers.
L'archive ouverte pluridisciplinaire HAL, est destinée au dépôt et à la diffusion de documents scientifiques de niveau recherche, publiés ou non, émanant des établissements d'enseignement et de recherche français ou étrangers, des laboratoires publics ou privés. 


\section{Rationnel pour une utilisation plus large de la Photochimiothérapie Extracorporelle chez l'enfant.}

Rationale for the use of Extracorporeal Photochemotherapy in children.

Etienne Merlin ${ }^{1,2}$, Justyna Kanold ${ }^{1,2,3}$, Dalil Hannani ${ }^{4}$, Daniel Terral ${ }^{1}$, Michel D’Incan ${ }^{5}$, François Deméocq $^{1,2,3}$.

1-CHU Clermont-Ferrand, Centre Régional de Cancérologie et Thérapie Cellulaire Pédiatrique, HôtelDieu, F-63001 Clermont-Ferrand, France.

2- Inserm, CIC 501, F-63003 Clermont-Ferrand, France.

3- Clermont Université, Univ Clermont1, Faculté de Médecine, F-63001 Clermont-Ferrand, France.

4- Inserm, U823, Immunobiologie et Immunothérapie des cancers, EFS La Tronche, F-38706 ; Univ Joseph Fourier, F-38041 Grenoble, France.

5. CHU Clermont-Ferrand, Service de dermatologie, Hôtel-Dieu, F-63001 Clermont-Ferrand, France.

Auteur correspondant :

Etienne Merlin

Centre Régional de Cancérologie et Thérapie Cellulaire Pédiatrique

Hôtel-Dieu

63058 Clermont-Ferrand cedex

Tél. : 0473750009

Fax : 0473750004

Courriel : e_merlin@chu-clermontferrand.fr

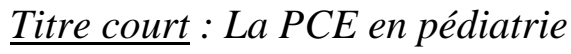

Mots clés : thérapie cellulaire ; psoralène ; aphérèse ; immunomodulation 


\section{Résumé}

Malgré l'apport des biothérapies, le traitement des maladies dysimmunitaires sévères et des conflits allogéniques de l'enfant reste difficile et entaché de nombreuses complications. Dans ce contexte, la photochimiothérapie extracorporelle (thérapie cellulaire qui repose sur l'effet immunomodulateur des cellules mononucléées du patient, prélevées par aphérèse et exposées ex vivo aux UVA en présence de psoralène) a l'avantage notable d'induire une tolérance immunitaire sans générer d'immunosuppression systémique ni de toxicité à court, moyen ou long terme. Cette immunomodulation fait intervenir notamment la génération de lymphocytes T régulateurs.

Malgré cela, la PCE est peu utilisée en raison de ses contraintes logistiques et du manque de données cliniques. Nous proposons une revue des indications reconnues et potentielles de la PCE en pédiatrie. Nous insistons sur la nécessité d'une évaluation clinique spécifique à l'enfant qui ne peut se concevoir sans la participation active des cliniciens pédiatres en particulier dans le domaine de la transplantation et des maladies auto-immunes et inflammatoires.

\section{Summary}

The management of immune diseases in children remains challenging although significant advances have been made. Beside the pharmacological approaches, Extracorporeal Photochemotherapy (ECP) is distinguishable by its ability to provide immunomodulation without immune suppression or toxicity. However, in practice this therapy is not widely used due to logistical issues and to the lack of robust clinical paediatric studies. Here we discuss the potential clinical applications of ECP in children; we insist on the need of a rigorous and specifically paediatric clinical evaluation of ECP. 


\section{INTRODUCTION}

Les pathologies dysimmunitaires sévères et conflits allogéniques occupent une place croissante dans la pathologie pédiatrique (1-3). Leur prise en charge médicamenteuse repose en grande partie sur les immunosuppresseurs, associant les traitements pharmacologiques « conventionnels » (corticothérapie, anti-calcineurine, anti-métabolites) et les biothérapies (anticorps monoclonaux, protéines recombinantes). L'objectif du traitement est d'obtenir une immunosuppression suffisante pour une efficacité thérapeutique au prix d'un risque infectieux et d'effets indésirables les plus faibles possibles. Les biothérapies ont profondément amélioré cette prise en charge, mais leur utilisation reste associée à un risque infectieux non-négligeable (4). D'autre part, leur efficacité n'est pas constante et les conséquences de leur utilisation à long terme sont encore mal connues chez l'enfant. La recherche de traitements non-toxiques reste donc un enjeu important.

La photochimiothérapie extracorporelle (PCE) ou photophérèse est une technique de thérapie cellulaire qui repose sur l'effet immunomodulateur des cellules mononucléées $(\mathrm{CMN})$ du patient, prélevées par aphérèse et exposées ex vivo aux UVA en présence d'une molécule photoactivable, le psoralène (5) (figure 1 et encadré). La réinjection des cellules traitées permet de réguler l'activité du système immunitaire sans entraîner d'immunosuppression généralisée ni d'effets indésirables (6). Actuellement l'indication prépondérante de la PCE, chez l'enfant comme chez l'adulte, est le traitement de la maladie du greffon contre l'hôte (GVHD pour Graft versus Host Disease) après allogreffe de cellules souches hématopoïétiques. Cependant, compte tenu de son mécanisme d'action et de son absence de toxicité, elle pourrait être proposée dans l'arsenal thérapeutique d'autres pathologies allogéniques ou dysimmunitaires de l'enfant (rejet de greffe, maladies inflammatoires du tube digestif, asthme sévère, rhumatismes inflammatoires etc.). 


\section{IMMUNOMODULATION PAR LA PCE}

\subsection{HISTORIQUE}

Avant la traversée du désert, les commerçants nomades du Sahara ingéraient des racines d'Ammi Majus, une plante herbacée commune du pourtour méditerranéen, pour accélérer leur bronzage et se protéger des brûlures solaires. L'ingestion de plantes photosensibilisantes (respectivement Psoralea Coryfolia et Ammi Majus) suivie d'exposition solaire volontaire à but thérapeutique est mentionnée dans le livre sacré indien Antharva Veda, et a été préconisée au 11e siècle de notre ère par Ibn El Bitar pour le traitement du vitiligo. Les psoralènes ont été extraits de l'Ammi Majus en 1911, et identifiés dans d'autres végétaux (persil, panais, céleri, fenouil, bergamote, citron, tilleul, gironfle) puis purifiés et utilisés en comprimés (Meladinine ${ }^{\odot}$ ) dès 1948, alors combinés à l'exposition solaire naturelle. A partir de 1965 l'irradiation UVA artificielle a été utilisée avec le psoralène (alors appliqué localement) pour le traitement du psoriasis, et c'est en 1974 que la Puvathérapie a été décrite dans sa forme actuelle (ingestion de 8-methoxypsoralène ou 8-MOP + exposition aux UVA contrôlée en cabine) pour le traitement du psoriasis puis, à partir de 1976, pour le lymphome T cutané (7).

Une forme particulière de lymphome $\mathrm{T}$ cutané est caractérisée par la présence dans la circulation sanguine de lymphocytes $\mathrm{T}$ du clone malin (syndrome de Sézary). C'est pour le traitement de cette maladie que Richard Edelson a inventé la PCE (ou photophérèse), en s'appuyant sur le phénomène de « vaccination $\mathrm{T} »$ décrit en 1981 (8) : l'injection intraveineuse de clones de lymphocytes T pathogènes inactivés ex vivo (par irradiation) induit in vivo une immunité dirigée contre ces clones (réponse antiidiotypique). Edelson a fait le postulat qu'en prélevant (par cytaphérèse), en traitant ex vivo (par Puvathérapie) puis en réinjectant les lymphocytes $\mathrm{T}$ du clone malin circulant, il devait être possible de déclencher une réponse anti-idiotypique contre les lymphocytes $\mathrm{T}$ du même clone infiltrant la peau. La publication des résultats en 1987 attestait de l'efficacité clinique du concept de vaccination T chez l'homme et de l'excellent profil de tolérance de la PCE, rapidement reconnue par la Food and Drug Administration comme un traitement de choix du syndrome de Sézary (9). 
La possibilité d'induire une immunité anti-idiotypique par la PCE laissait alors espérer un traitement immunomodulateur aux allures de panacée: permettant de traiter sélectivement les clones de lymphocytes $\mathrm{T}$ pathogènes, et dépourvu de toxicité. La PCE a alors fait l'objet d'une multitude de publications de cas isolés ou de petites séries de patients : psoriasis, complexe cutané du SIDA, rhumatismes inflammatoires chroniques, hépatite virale $\mathrm{C}$, sclérose en plaque, rejet de greffe, maladie du greffon contre l'hôte (GVHD) etc. Quelques études prospectives contrôlées ont permis de conclure à l'efficacité de la PCE notamment pour la prévention du rejet de greffe cardiaque (10) et pour la prévention de la sténose coronaire après angioplastie (11) ; à son inefficacité pour la prévention des poussées de sclérose en plaque (12); et à un effet significatif mais «cliniquement insuffisant » pour la sclérodermie systémique (13) (sauf utilisée précocement après le début des signes, où le bénéfice clinique est alors important) et pour le diabète auto-immun (14).

Actuellement, la PCE est un traitement de routine utilisé quotidiennement dans les unités d'aphérèse thérapeutique, essentiellement pour le traitement du lymphome T cutané, de la GVHD aiguë et chronique, et de façon plus anecdotique du rejet de greffe d'organe, de la fibrose néphrogénique et de pathologies auto-immunes en particulier dermatologiques (lichen plan érosif, maladies bulleuses, lupus cutané, etc.).

\subsection{TECHNIQUE}

La PCE comprend trois étapes : 1/ prélèvement des cellules mononucléées (CMN) du patient par cytaphérèse (séparation des cellules par centrifugation au cours d'une circulation extracorporelle) 2/ photosensibilisation et irradiation UVA des cellules prélevées 3/ retour au patient par voie veineuse (figure 1). Une séance dure entre 2,5 et 3 heures. Le rythme des séances dépend des indications, entre trois séances hebdomadaires et une séance mensuelle. Le prélèvement est l'étape clé : il représente la seule vraie contrainte du traitement, et conditionne à la fois la qualité du produit cellulaire (richesse et pureté) et la tolérance du patient (durée de la séance, déplétion plaquettaire et en hémoglobine, vécu des 
séances). Le prélèvement ne pose pas de difficulté majeure chez l'adulte ; l'exercice pédiatrique est rendu plus difficile par les contraintes techniques, physiologiques et psychologiques propres à l'enfant. Actuellement, deux techniques de PCE sont disponibles : la technique «en système dissocié » (dite «Vilber-Lourmat») et la technique « en ligne»(dite «Therakos»). Le système dissocié peut être proposé aux enfants de faible poids (à partir de $10 \mathrm{~kg}$ ), permet un prélèvement de très bonne qualité et laisse la possibilité de préparer le produit cellulaire «à la carte », mais requiert l'intervention d'un laboratoire de thérapie cellulaire (15). Le système en ligne est applicable à partir de $20 \mathrm{~kg} \mathrm{ou} 40 \mathrm{~kg}$ selon le dispositif utilisé ; il ne nécessite pas de laboratoire de thérapie cellulaire, mais ne permet pas de manipulation ex vivo du produit cellulaire (figure 2).

\subsection{MECANISME D’ACTION}

\subsubsection{Généralités}

L'action de la PCE repose sur la neutralisation des clones de lymphocytes T pathogènes, par l'induction d'une immunité dirigée contre ces clones (immunité anti-clonotypique ou anti-idiotypique) (6). Ceci explique son effet dans le traitement du lymphome (clone malin), des conflits allogéniques (clones alloréactifs) et des maladies auto-immunes (clones auto-réactifs). La spécificité vis-à-vis d'un clone peut s'expliquer par le fait que les corps apoptotiques issus de lymphocytes activés ont un phénotype « régulateur » (augmentation du CD5, diminution du CD28 et du CD86) (16). Cette neutralisation élective aboutit à une «délétion clonale» mais préserve la capacité du système immunitaire à répondre à de nouveaux antigènes ou à la réintroduction d'antigènes connus (vaccinaux) (17). Il s'agit donc d'une immunomodulation qui ne relève pas d'un mécanisme immunosuppresseur généralisé.

Les mécanismes qui sous-tendent cette neutralisation impliquent une coopération entre lymphocytes, monocytes et cellules dendritiques. Il s'agit probablement de l'amplification d'un ou plusieurs mécanismes physiologiques de régulation immunitaire déclenchés par la coexistence d'un stress oxydatif et d'une population lymphocytaire T monoclonale ou oligoclonale apoptotique, comme c'est le 
cas par exemple lors de la réaction inflammatoire. Les lymphocytes T régulateurs CD4+CD25+FoxP3+ (T reg) et l'interleukine 10 sont essentiels à l'action de la PCE (18).

Cependant, malgré de nombreuses recherches, les mécanismes d'action de la PCE ne sont pas complètement élucidés. En raison de leur complexité, et vraisemblablement parce qu'il existe plusieurs phénomènes aboutissant à la neutralisation des clones lymphocytaires T pathogènes. Egalement parce que ces mécanismes dépendent probablement de l'état du clone traité (malignité, prolifération, profil Th1/Th2, etc.). Enfin parce que les études chez l'homme sont difficiles à interpréter en raison de l'hétérogénéité des patients, des traitements concomitants, et de l'absence fréquente de groupe contrôle comparable. Nous ne présenterons ici que les données les mieux documentées sur les mécanismes d'action de la PCE, qui sont résumés dans la figure 3.

\subsubsection{Effets de l'association 8-MOP + UVA}

Sous l'effet du rayonnement UVA (365 nm), le 8-MOP se lie aux biomolécules environnantes (ADN, protéines et lipides), et constitue des ponts entre les bases thymidine de l'ADN (19). En fonction de la dose, les lésions de l'ADN peuvent entraîner des modifications transcriptionnelles, l'apoptose ou la nécrose cellulaire.

Il existe d'autres agents photosensibilisants dont l'action repose sur les mêmes mécanismes que le 8MOP. Seules leurs caractéristiques pharmacocinétiques sont différentes, et moins avantageuses. Le seul actuellement utilisé est l'amotosalène, un psoralène de synthèse combiné aux UVA pour l'inactivation des agents infectieux contaminant potentiellement les produits sanguins (système Intercept) (20).

\subsubsection{Interactions cellulaires et modifications de l'environnement}

Les lésions de l'ADN déclenchent l'apoptose lymphocytaire, par la voie des caspases et de Bcl2 (21). Les lymphocytes apoptotiques sont phagocytés par les cellules présentatrices d'antigène (CPA) contenues dans le produit cellulaire (monocytes et cellules dendritiques rendues immatures par le stress oxydatif, et dont l'apoptose est plus lente que celle des lymphocytes $\mathrm{T}$ (22)) et après réinjection par les 
CPA non-traitées. Dans le cas des conflits allogéniques, cette phagocytose entraîne une modification de l'environnement cellulaire, en particulier la production d'interleukine 10 et l'inhibition du TNF $\alpha$ par les cellules monocytaires et dendritiques (23). Cette interaction rend les cellules dendritiques «tolérogènes », capables de déclencher la neutralisation ou la destruction du clone phagocyté. La neutralisation fait intervenir les lymphocytes CD4 régulateurs (Treg CD4+CD25+FoxP3+) et la sécrétion d'interleukine 10 (18); le rôle de la molécule inhibitrice CTLA4 et des lymphocytes suppresseurs CD8+ est suspecté mais moins clairement établi (6).

\subsection{ACTIVITE FRANÇAISE (DONNEES DE LA SOCIETE FranÇAISE DE PCE)}

En 2008, selon les données de la Société Française de Photochimiothérapie Extracorporelle (SFPCE), la technique était réalisée dans 15 centres français dont 6 Etablissements Français du Sang et 9 établissements de soins. Entre 2004 et 2008, le nombre de séances annuelles est passé de 2051 (131 patients) à 2846 (219 patients dont 19 enfants). Les indications majoritaires chez l'adulte étaient la GVHD, le lymphome cutané et le rejet d'organe solide. La part de la GVHD a augmenté régulièrement depuis 2004 (figure 4). Les centres ayant une expérience pédiatrique sont : Besançon, Clermont-Fd, Grenoble, Marseille, Nancy, Nantes, Nice, Paris Debré, Paris St-Louis, et Rouen.

\section{DONNEES CLINIQUES}

Les données sur la PCE concernent les conflits allogéniques, les maladies auto-immunes ou inflammatoires et les proliférations malignes clonales $\mathrm{T}$ à cinétique lente (ces dernières ne concernent pas l'enfant et ne seront pas discutées ici). Les données pédiatriques publiées ne concernent que la GVHD et le diabète de type 1 ; nous envisagerons ensuite les pathologies pour lesquelles il existe un rationnel clinique ou fondamental qui justifie l'évaluation de la PCE chez l'enfant et l'adolescent. 


\subsection{DONNEES PEDIATRIQUES}

\subsubsection{GVHD}

Après une allogreffe de cellules souches hématopoïétiques $(\mathrm{CSH})$, la réaction allogénique du greffon contre l'hôte est à l'origine d'une expression clinique délétère (la GVHD pour Graft Versus Host Disease ou Maladie du Greffon contre l'Hôte) et d'un effet bénéfique par l'éradication des cellules malignes (effet du greffon contre la leucémie $=$ GVL ou contre la tumeur $=$ GVT $)$.

Dans le cas des maladies malignes, le but de l'allogreffe est d'obtenir un effet GVL sans manifestation de GVHD. Or, cette dernière survient après 30 à $50 \%$ des 300 allogreffes pédiatriques réalisées annuellement en France (24). Elle expose à un risque important de mortalité et d'altération de la qualité de vie à court, moyen et long terme, en partie à cause des traitements immunosuppresseurs qu'elle nécessite (25). Chez l'enfant de moins de 5 ans, 45\% des greffes sont réalisées en traitement curatif d'une maladie non-maligne où la réaction allogénique n'a pas d'intérêt thérapeutique (3). Dans cette situation encore plus que dans le cas des maladies malignes, tout doit être mis en œuvre pour prévenir et traiter les complications dues à la GVHD et à ses traitements.

L'originalité de la PCE tient à sa capacité à traiter la GVHD sans toxicité et tout en préservant l'effet GVL. Les données pédiatriques publiées concernent près de 180 patients. Elles émanent de 7 équipes dans le monde (essentiellement italiennes et françaises) (26-32), et sont résumées dans le tableau 1. Ces cohortes historiques non-contrôlées doivent être considérées sous réserve des difficultés méthodologiques inhérentes à ce type d'études. Néanmoins, les taux de réponse très encourageants font de la PCE un traitement de choix de la GVHD en cas d'efficacité insuffisante de la corticothérapie (33). Les indications de PCE sont les mêmes que chez l'adulte :

- GVHD réfractaire au traitement de 1è et/ou de 2è ligne

- effets indésirables sévères des immunosuppresseurs

- processus évolutif ou infectieux nécessitant une diminution rapide de l'immunosuppression. 
Dans notre centre, 35 enfants ont été traités depuis 1996. Avec l'expérience, l'indication de la PCE a été posée de plus en plus tôt dans l'évolution de la GVHD ; elle est maintenant systématiquement envisagée en l'absence de réponse à la corticothérapie administrée pendant 2 à 3 jours (GVHD aiguë) ou 30 jours (GVHD chronique).

\subsubsection{Diabète de type 1.}

La préservation de la sécrétion résiduelle d'insuline est un enjeu très important pour le devenir à long terme des patients atteints de diabète de type 1. En 2000, une équipe suédoise a publié un essai contrôlé randomisé de PCE contre placebo (aphérèses «factices ») chez 40 enfants au diagnostic de diabète de type 1 (14). Cet de PCE contrôlé randomisé contre placebo a été conduit chez des patients naïs de tout traitement immunosuppresseur, dans une pathologie auto-immune caractérisée par des antigènes cibles peu nombreux et bien identifiés ; il s'agit donc d'un excellent modèle d'étude. Cet essai a montré que la PCE réduisait les besoins en insuline à moyen terme (3 ans), et induisait une tolérance spécifique vis-àvis de l'auto-antigène GAD (glutamic acide decarboxylase) via les molécules CTLA4 et les Treg (34). Ceci atteste de la réalité du phénomène de neutralisation clonale dans le contexte d'une maladie autoimmune chez l'homme.

Dans cet essai, les patients du groupe PCE ont reçu 1 séance hebdomadaire pendant 6 semaines. Lors des 3 ans de suivi, les taux d'hémoglobine glyquée étaient similaires dans les 2 groupes. En l'absence de tests de stimulation, les patients du groupe PCE avaient une excrétion urinaire de peptide C plus élevée et des taux sanguins plus élevés que les témoins, mais ces différences n'étaient pas statistiquement significatives. Les besoins en insuline des patients traités étaient moins élevés que ceux des témoins. Aucune donnée n'était rapportée sur le suivi à plus long terme des patients, ni sur l'incidence des accidents aigus.

Dans ces conditions, les auteurs ont estimé que des connaissances plus approfondies sur les effets de la PCE étaient nécessaires avant qu'elle puisse être considérée comme un traitement systématique, eu 
égard à son coût. Cependant, compte tenu de l'importance des complications tardives du diabète et de l'innocuité de ce traitement peu intensif un recul plus long serait nécessaire.

\subsubsection{Expérience personnelle de la PCE chez l'enfant (hors GVHD)}

Nous avons utilisé la PCE dans les indications suivantes chez l'enfant. Chez une patiente de 8 ans atteinte d'hémosidérose pulmonaire idiopathique corticodépendante, l'adjonction de la PCE a permis de diminuer la dose minimale de prednisone nécessaire à la prévention des poussées. Nous avons traité deux patientes atteintes de sclérodermie monomélique sévère: la première (alors âgée de 18 ans) plusieurs années après le diagnostic, alors que la sclérose cutanée était sévère, et responsable de rétractions articulaires et d'une hypotrophie du membre. Nous avons observé une modification de la pigmentation cutanée, mais pas d'amélioration significative de l'atteinte scléreuse. La seconde patiente, âgée de 5 ans, a été traitée par PCE et methotrexate 3 mois après le diagnostic de sclérodermie monomélique étendue à l'ensemble du membre inférieur, associée à une atteinte déformante du 5è rayon de la main et trois plaques de morphée. La sclérose cutanée et les plaques de morphée ont complètement disparu, la peau gardant une hyperpigmentation d'allure séquellaire. Après un an de traitement, une atrophie du tissu adipeux est apparue mais la fonctionnalité du membre est normale. Un enfant de 10 ans a été traité pour une dermatopolymyosite dont les signes cutanés persistaient après 6 semaines de corticothérapie ; l'érythème héliotrope a disparu en 2 semaines après le début de la PCE. Celle-ci a été poursuivie pendant 18 mois associée à la décroissance de la corticothérapie. Le patient est actuellement asymptomatique sans traitement avec un an de recul.

\subsection{APPLICATIONS POSSIBLES EN PEDIATRIE}

\subsubsection{Transplantation d'organe}

En 2008, 196 enfants de moins de 18 ans ont reçu une transplantation d'organe en France (3). Malgré des progrès continus, la transplantation d'organe chez l'enfant reste une situation très difficile en partie à cause de l'équilibre précaire entre efficacité et complications des immunosuppresseurs. Le rejet aigu ou 
chronique reste la difficulté majeure. L'enfant greffé va donc être exposé pendant une durée extrêmement longue aux immunosuppresseurs, et de ce fait à leur toxicité «consentie » (augmentation du risque infectieux et de lymphoprolifération) et «non-consentie» (troubles métaboliques, endocriniens etc.). La survie à 5 ans des enfants après une greffe de poumons est de $50 \%$; elle est de $67 \%$ après une transplantation cardiaque. Dix ans après une transplantation rénale, $95 \%$ des enfants sont vivants mais la probabilité de survie d'un greffon rénal est de $68 \%$ à 10 ans (3). Les rejets et les infections sont la cause de la majorité des complications, perte du greffon ou décès (35).

Chez l'adulte, l'efficacité de la PCE est très bien documentée pour la prévention et le traitement du rejet cardiaque, et de façon moins solide pour le rejet rénal, pulmonaire et hépatique. Actuellement chez l'adulte deux indications de la PCE sont reconnues (36):

- rejet malgré une immunosuppression bien conduite

- nécessité de diminuer les traitements immunosuppresseurs (infection évolutive ou menaçante, syndrome lymphoprolifératif, effet indésirable spécifique)

Chez l'enfant, il conviendrait d'en ajouter une troisième : «prévention des complications à long terme et des séquelles des traitements immunosuppresseurs ». Nous pensons qu'un effort de sensibilisation doit être effectué en direction des pédiatres greffeurs, pour que soit réalisée chez l'enfant l'évaluation clinique de la PCE telle qu'elle a apporté la preuve de son intérêt dans le domaine de la transplantation chez l'adulte.

\subsubsection{Maladies auto-immunes et inflammatoires (tableau 2).}

Le mécanisme d'action le mieux documenté de la PCE est l'induction de lymphocytes T régulateurs (Treg) de phénotype CD4+CD25+FoxP3+. Ces lymphocytes ont la capacité de réguler le système immunitaire pour prévenir l'émergence de maladies auto-immunes. L'importance de cette fonction régulatrice est illustrée par la gravité du syndrome IPEX (déficit congénital en Treg dû à une mutation de FoxP3) (37). Or, l'implication d'un défaut de la fonction des Treg a été montrée dans de nombreuses 
pathologies auto-immunes ou inflammatoires, en particulier l'asthme, les maladies rhumatismales et la maladie de Crohn (38).

Les maladies pour lesquelles l'efficacité clinique de la PCE a été rapportée chez l'adulte recoupent en partie celles impliquant un dysfonctionnement des T reg (tableau 2): maladie de Crohn (36, 39), polyarthrite rhumatoïde $(15,40,41)$ et rhumatisme psoriasique $(42)$, dermatite atopique $(43)$; la PCE a également été utilisée avec succès pour le traitement du lichen plan érosif $(44,45)$ et de certaines connectivites telles que la sclérodermie $(13,46,47)$ ou la dermatopolymyosite (48).

\subsection{TOLERANCE ET REALISATION PRATIQUE CHEZ L'ENFANT}

\subsubsection{Effets indésirables}

A ce jour, aucune complication sévère et aucun effet indésirable à long terme n'ont été imputés à la PCE. On recense un cas de chorio-rétinite chez un adulte (49), et un cas d'hyperéosinophilie importante chez un enfant (données non publiées). En dehors de ces cas exceptionnels, les effets indésirables cliniques sont rares et bénins. Dans les études contrôlées, la fréquence des bactériémies n’est pas augmentée chez les patients traités par PCE.

L'effet indésirable le plus constant est une baisse du taux d'hémoglobine d'environ $1 \mathrm{~g} / \mathrm{dl}$ par séance. Elle est due à l'hémolyse mécanique et à l'hémodilution. La cytaphérèse est responsable d'une diminution de 15 à $17 \%$ du taux de plaquettes (26). En dehors de l'allogreffe de cellules souches hématopoïétiques, les transfusions sont exceptionnelles.

\subsubsection{Réalisation pratique de la PCE chez l'enfant}

A condition de leur porter une grande attention, les trois principales difficultés propres à l'enfant sont faciles à prévenir et ne posent pas de difficulté majeure.

L'abord veineux est le problème le plus important, mais en l'absence d'atteinte cutanée sévère un traitement sur voie veineuse périphérique exclusive ou combinée au cathéter central déjà en place (patients d'onco-hématologie) est généralement possible. La circulation extracorporelle expose à un 
risque d'hypovolémie, d'hypocalcémie (due au citrate qui est l'anticoagulant le plus fréquemment utilisé) et d'hypothermie. Nous avons publié en 2007 des recommandations spécifiques pour la prévention de ces complications (26). En suivant ces recommandations simples, sur plus de 1000 séances chez 40 enfants nous n'avons jamais rencontré de complication y compris chez les enfants de moins de $15 \mathrm{~kg}$.

La contrainte que représente la PCE est immédiate et facilement identifiable, alors que ses bénéfices sont différés (l'effet du traitement n'est pas immédiat ; il faut entre 7 jours et plusieurs semaines pour en voir les effets apparaître dans le cas de la GVHD). Cette différence par rapport aux immunosuppresseurs (dont les bénéfices sont rapides et les contraintes différées) explique que le « ras-le-bol » du traitement en général s'exprime volontiers sous la forme d'une stigmatisation de la PCE. Il est donc fondamental, pour une bonne acceptation du traitement, que l'enfant et sa famille aient bien compris les enjeux de la PCE pour la prévention des complications à moyen et à long terme. Il faut également veiller au confort de l'enfant pendant l'aphérèse, qui peut se faire sur les genoux des parents, dans la chambre d'hospitalisation y compris sous flux laminaire ou en environnement protégé. Enfin, il est préférable de différer une séance de 24 heures et de bien réexpliquer les tenants et aboutissants du traitement plutôt que d'imposer coûte que coûte l'aphérèse à un enfant en opposition.

Les conditions de l'aphérèse (immobilité, durée, bruit, abords veineux) rendent compte de la perception souvent exagérée qu'en ont les cliniciens (y compris pédiatres) chez l'enfant. Nous insistons sur le fait que, sous réserve d'une bonne information et d'une prise en charge adaptée à l'âge de l'enfant et à sa famille, la PCE est un traitement bien toléré.

\section{QUELLE PLACE POUR LA PCE EN PEDIATRIE?}

L'effet immunomodulateur de la PCE est cliniquement significatif, dépourvu de toxicité et d'immunosuppression généralisée. En cela, la PCE diffère de la quasi-totalité des traitements proposés aux enfants atteints de pathologies à médiation immunitaire. La seule limite à son utilisation est donc 
liée aux contraintes logistiques et de temps, qui doivent être mises en balance avec son double bénéfice : effet thérapeutique propre, et diminution des traitements immunosuppresseurs.

La mesure de ce rapport bénéfice / contrainte ne peut pas être extrapolée des données adultes, et nécessite une évaluation clinique spécifique à l'enfant. Or, la PCE est une thérapie cellulaire dont l'évaluation et la promotion chez l'enfant ne dépendent pas de l'industrie, mais des cliniciens et des unités de thérapie cellulaire. Son utilisation thérapeutique raisonnée en pédiatrie nécessite donc que cette évaluation soit entreprise par les cliniciens pédiatres. Sous le mandat et à la demande de la Société Française de Greffe de Moelle et Thérapie Cellulaire (SFGM-TC), nous avons ouvert en France en 2009 le premier protocole prospectif d'évaluation de la PCE en traitement de la GVHD réfractaire chez l'enfant (50). Ce protocole est un premier pas important, puisqu'il a conduit à l'obtention pour les centres participants d'une autorisation de l'Agence Française de Sécurité Sanitaire et des Produits de Santé (AFSSaPS) pour le procédé PCE, et au rapprochement entre eux des centres engagés dans l'évaluation clinique de la PCE chez l'enfant. Ce premier essai devrait donc faciliter la mise en place d'essais cliniques pour d'autres pathologies. Les maladies que nous identifions en particulier sont la maladie de Crohn, l'arthrite chronique juvénile et les sclérodermies.

\section{CONCLUSION}

La PCE est un traitement immunomodulateur puissant et dénué de toxicité, mais dont l'utilisation est limitée par la lourdeur. Pourtant, dans une équipe entraînée à la pratique de l'aphérèse chez l'enfant, ce traitement est faisable et bien toléré y compris chez les enfants de faible poids (à partir de $10 \mathrm{~kg}$ ). Son avantage majeur - induction d'une tolérance immunitaire sans immunosuppression ni toxicité - trouve chez l'enfant un intérêt particulier. A ce jour, les données cliniques pédiatriques sont insuffisantes et ce traitement est probablement sous-exploité. Ceci justifie que soit menée une double démarche de sensibilisation et d'évaluation, en particulier dans le domaine de la transplantation d'organe et des maladies dysimmunitaires et inflammatoires sévères. 


\section{Références}

1. Malaty HM, Fan X, Opekun AR, Thibodeaux C, Ferry GD. Rising incidence of inflammatory bowel disease among children: a 12-year study. J Pediatr Gastroenterol Nutr;50(1):27-31.

2. Borchers AT, Uibo R, Gershwin ME. The geoepidemiology of type 1 diabetes. Autoimmun Rev 2009.

3. Agence de la Biomédecine, rapport 2008, disponible sur http://www.agencebiomedecine.fr/professionnels/rapport-annuel.html.

4. Bader-Meunier B, Quartier P. Nouveautés pharmaceutiques dans l'arthrite juvénile idiopathique. Arch Pediatr 2009;16(12):1607-11.

5. Kanold J, Halle P, Tuppin P, Demeocq F. Thérapie cellulaire dans le traitement du cancer de l'enfant. Arch Pediatr 2004;11(2):130-43.

6. Marshall SR. Technology insight: ECP for the treatment of GvHD--can we offer selective immune control without generalized immunosuppression? Nat Clin Pract Oncol 2006;3(6):302-14.

7. Heshmati F, Andreu G. Extracorporeal photochemotherapy: a historical perspective. Transfus Apher Sci 2003;28(1):25-34.

8. Ben-Nun A, Wekerle H, Cohen IR. Vaccination against autoimmune encephalomyelitis with Tlymphocyte line cells reactive against myelin basic protein. Nature 1981;292(5818):60-1.

9. Edelson R, Berger C, Gasparro F, et al. Treatment of cutaneous T-cell lymphoma by extracorporeal photochemotherapy. Preliminary results. N Engl J Med 1987;316(6):297-303.

10. Barr ML, Meiser BM, Eisen HJ, et al. Photopheresis for the prevention of rejection in cardiac transplantation. Photopheresis Transplantation Study Group. N Engl J Med 1998;339(24):1744-51.

11. Bisaccia E, Palangio M, Gonzalez J, Klainer AS, Banas JS. Photopheresis. Therapeutic potential in preventing restenosis after percutaneous transluminal coronary angioplasty. Am J Cardiovasc Drugs 2003;3(1):43-51.

12. Rostami AM, Sater RA, Bird SJ, et al. A double-blind, placebo-controlled trial of extracorporeal photopheresis in chronic progressive multiple sclerosis. Mult Scler 1999;5(3):198-203.

13. Knobler R, French L, Kim Y, et al. A randomized, double-blind, placebo-controlled trial of photopheresis in systemic sclerosis. J Am Acad Dermatol 2006;54(5):793-9.

14. Ludvigsson J, Samuelsson U, Ernerudh J, Johansson C, Stenhammar L, Berlin G. Photopheresis at onset of type 1 diabetes: a randomised, double blind, placebo controlled trial. Arch Dis Child 2001;85(2):149-54.

15. Andreu G, Leon A, Heshmati F, et al. Extracorporeal photochemotherapy: evaluation of two techniques and use in connective tissue disorders. Transfus Sci 1994;15(4):443-54.

16. Stadler K, Frey B, Munoz LE, et al. Photopheresis with UV-A light and 8-methoxypsoralen leads to cell death and to release of blebs with anti-inflammatory phenotype in activated and non-activated lymphocytes. Biochem Biophys Res Commun 2009;386(1):71-6.

17. Suchin KR, Cassin M, Washko R, et al. Extracorporeal photochemotherapy does not suppress Tor B-cell responses to novel or recall antigens. J Am Acad Dermatol 1999;41(6):980-6.

18. Maeda A. Extracorporeal photochemotherapy. J Dermatol Sci 2009;54(3):150-6.

19. Gasparro FP, Song J, Knobler RM, Edelson RL. Quantitation of psoralen photoadducts in DNA isolated from lymphocytes treated with 8-methoxypsoralen and ultraviolet A radiation (extracorporeal photopheresis). Curr Probl Dermatol 1986;15:67-84.

20. Amotosalen: Allogeneic Cellular Immunotherapies system, INTERCEPT Plasma System, INTERCEPT Platelet System, S 59. BioDrugs 2003;17(1):66-8.

21. Szodoray P, Papp G, Nakken B, Harangi M, Zeher M. The molecular and clinical rationale of extracorporeal photochemotherapy in autoimmune diseases, malignancies and transplantation. Autoimmun Rev 2010;9(6):459-64. 
22. Hannani D, Gabert F, Laurin D, et al. Photochemotherapy induces the apoptosis of monocytes without impairing their function. Transplantation 2010; in press.

23. Craciun LI, Stordeur P, Schandene L, et al. Increased production of interleukin-10 and interleukin-1 receptor antagonist after extracorporeal photochemotherapy in chronic graft-versus-host disease. Transplantation 2002;74(7):995-1000.

24. Ferry C, Gemayel G, Rocha V, et al. Long-term outcomes after allogeneic stem cell transplantation for children with hematological malignancies. Bone Marrow Transplant 2007;40(3):21924.

25. Michel G, Bordigoni P, Simeoni MC, et al. Health status and quality of life in long-term survivors of childhood leukaemia: the impact of haematopoietic stem cell transplantation. Bone Marrow Transplant 2007;40(9):897-904.

26. Kanold J, Merlin E, Halle P, et al. Photopheresis in pediatric graft-versus-host disease after allogeneic marrow transplantation: clinical practice guidelines based on field experience and review of the literature. Transfusion 2007;47(12):2276-89.

27. Messina C, Locatelli F, Lanino E, et al. Extracorporeal photochemotherapy for paediatric patients with graft-versus-host disease after haematopoietic stem cell transplantation. Br J Haematol 2003;122(1):118-27.

28. Berger M, Pessolano R, Albiani R, et al. Extracorporeal photopheresis for steroid resistant graft versus host disease in pediatric patients: a pilot single institution report. J Pediatr Hematol Oncol 2007;29(10):678-87.

29. Calore E, Calo A, Tridello G, et al. Extracorporeal photochemotherapy may improve outcome in children with acute GVHD. Bone Marrow Transplant 2008;42(6):421-5.

30. Duzovali O, Chan KW. Intensive extracorporeal photochemotherapy in pediatric patients with chronic graft-versus-host disease (cGVHD). Pediatr Blood Cancer 2007;48(2):218-21.

31. Perseghin P, Dassi M, Balduzzi A, Rovelli A, Bonanomi S, Uderzo C. Mononuclear cell collection in patients undergoing extra-corporeal photo-chemotherapy for acute and chronic graft-vs.host-disease (GvHD): comparison between COBE Spectra version 4.7 and 6.0 (AutoPBSC). J Clin Apher 2002;17(2):65-71.

32. Gonzalez-Vicent M, Ramirez M, Perez A, Lassaletta A, Sevilla J, Diaz MA. Extracorporeal photochemotherapy for steroid-refractory graft-versus-host disease in low-weight pediatric patients. Immunomodulatory effects and clinical outcome. Haematologica 2008;93(8):1278-80.

33. Kanold J, Messina C, Halle P, et al. Update on extracorporeal photochemotherapy for graftversus-host disease treatment. Bone Marrow Transplant 2005;35 Suppl 1:S69-71.

34. Jonson CO, Pihl M, Nyholm C, Cilio CM, Ludvigsson J, Faresjo M. Regulatory T cell-associated activity in photopheresis-induced immune tolerance in recent onset type 1 diabetes children. Clin Exp Immunol 2008;153(2):174-81.

35. Tangeraas T, Bjerre A, Lien B, et al. Long-term outcome of pediatric renal transplantation: the Norwegian experience in three eras 1970-2006. Pediatr Transplant 2008;12(7):762-8.

36. Abreu MT, von Tirpitz C, Hardi R, et al. Extracorporeal photopheresis for the treatment of refractory Crohn's disease: results of an open-label pilot study. Inflamm Bowel Dis 2009;15(6):829-36.

37. Marabelle A, Meyer M, Demeocq F, Lachaux A. [From Ipex to foxp3: a new contribution of pediatrics to the understanding of the immune system]. Arch Pediatr 2008;15(1):55-63.

38. Brusko TM, Putnam AL, Bluestone JA. Human regulatory T cells: role in autoimmune disease and therapeutic opportunities. Immunol Rev 2008;223:371-90.

39. Reinisch W, Nahavandi H, Santella R, et al. Extracorporeal photochemotherapy in patients with steroid-dependent Crohn's disease: a prospective pilot study. Aliment Pharmacol Ther 2001;15(9):131322.

40. Malawista S, Trock D, Edelson R. Treatment of rheumatoid arthritis by extracorporeal photochemotherapy. A pilot study. Arthritis Rheum 1991;34(6):646-54. 
41. Hilliquin P, Andreu G, Heshmati F, Menkès C. Traitement de la polyarthrite rhumatoïde réfractaire par photochimiothérapie extra-corporelle. Rev Rhum Ed Fr 1993 60(2):125-30. .

42. Vahlquist C, Larsson M, Ernerudh J, Berlin G, Skogh T, Vahlquist A. Treatment of psoriatic arthritis with extracorporeal photochemotherapy and conventional psoralen-ultraviolet A irradiation. Arthritis Rheum 1996 39(9):1519-23.

43. Sand M, Bechara FG, Sand D, et al. Extracorporeal photopheresis as a treatment for patients with severe, refractory atopic dermatitis. Dermatology 2007;215(2):134-8.

44. Guyot AD, Farhi D, Ingen-Housz-Oro S, et al. Treatment of refractory erosive oral lichen planus with extracorporeal photochemotherapy: 12 cases. Br J Dermatol 2007;156(3):553-6.

45. Bécherel $\mathrm{P}$, Bussel $\mathrm{A}$, Chosidow $\mathrm{O}$, Rabian $\mathrm{C}$, Piette J, Francès C. Extracorporeal photochemotherapy for chronic erosive lichen planus. Lancet 1998;351(9105):805.

46. Rook A, Freundlich B, Jegasothy B, et al. Treatment of systemic sclerosis with extracorporeal photochemotherapy. Results of a multicenter trial. Arch Dermatol 1992;128(3):337-46.

47. Enomoto D, Mekkes J, Bossuyt P, et al. Treatment of patients with systemic sclerosis with extracorporeal photochemotherapy (photopheresis). J Am Acad Dermatol 1999;41(6):915-22.

48. Cribier B, Faradji T, Le Coz C, Oberling F, Grosshans E. Extracorporeal photochemotherapy in systemic sclerosis and severe morphea. Dermatology 1995;191(1):25-31.

49. Vagace JM, Gervasini G, Morais F, et al. Retinal toxic reactions following photopheresis. Arch Dermatol 2007;143(5):622-5.

50. Merlin E, disponible sur http://clinicaltrials.gov/ct2/show/NCT00824954?term=merlin\&rank=7.

\section{Conflits d'intérêt : aucun}

